IP Periodica Polytechnica Chemical Engineering

61(4), pp. 258-263, 2017

https://doi.org/10.3311/PPch.11087

Creative Commons Attribution (i)

RESEARCH ARTICLE

\section{Attempted Diels-Alder Reactions on Vindoline Derivatives}

\author{
Szabolcs Mayer ${ }^{1}$, Péter Keglevich ${ }^{1 *}$, Péter Ábrányi-Balogh ${ }^{2}$,
} Áron Szigetvári ${ }^{3}$, Miklós Dékány ${ }^{3}$, Csaba Szántay Jr. ${ }^{3}$, László Hazai ${ }^{1}$

Received 30 May 2017; accepted after revision 18 August 2017

\begin{abstract}
The Diels-Alder reaction of vindoline and methyl vinyl ketone resulted in a Friedel-Crafts reaction product. In the reaction between the ortho-quinone derivative of vindoline and $N$-phenylmaleimide, two anomalous products were obtained, a vindoline dimer, and a condensed vindoline derivative.
\end{abstract}

\section{Keywords}

vindoline, Friedel-Crafts reaction, N-phenylmaleimide, condensation, rearrangement

\footnotetext{
${ }^{1}$ Department of Organic Chemistry and Technology,

Faculty of Chemical Technology and Biotechnology, Budapest University of Technology and Economics,

H-1111 Budapest, Gellért tér 4., Hungary

${ }^{2}$ Institute of Organic Chemistry,

Research Centre for Natural Sciences,

Hungarian Academy of Sciences,

H-1519 Budapest, P. O. Box 286, Hungary

${ }^{3}$ Spectroscopic Research Department, Gedeon Richter Plc.,

H-1475 Budapest 10, P. O. Box 27, Hungary

${ }^{*}$ Corresponding author, e-mail: pkeglevich@mail.bme.hu
}

\section{Introduction}

The "dimeric" alkaloids vinblastine (1) and vincristine (2) (Fig. 1), which comprise the "monomeric" units catharanthine (3) and vindoline (4) were isolated from the Madagascar periwinkle, Catharanthus roseus. These types of compounds have been known as anticancer agents for more than forty years $[1,2]$.

Vinblastine (1) has an antimicrotubule effect used for treating certain kinds of cancer, including Hodgkin's lymphoma, nonsmall-cell lung cancer, breast cancer, head and neck cancer and testicular cancer. Vincristine (2), the $\mathrm{N}$-formyl derivative of vinblastine (1) can be used in various types of chemotherapy. Its main uses are in the treatment of non-Hodgkin's and Hodgkin's lymphoma, acute lymphoblastic leukaemia, and nephroblastoma.

In the course of the synthetic investigations of dimeric Vinca alkaloids, various methods have been elaborated to synthesize new derivatives of vinblastine (1) and vincristine (2) with improved therapeutic effect exhibiting a higher selectivity and a lower toxicity $[3,4]$.

The Diels-Alder reaction was applied in the total synthesis of vinblastine analogues [5], in the enantioselective total synthesis of the akuammiline alkaloid (-)-vincorine [6], and the Strychnos alkaloid (+)-minfiensine [7], as well as in the synthesis of some Aspidosperma alkaloids [8]. Rearrangement through a retro Diels-Alder reaction was observed during the investigations of (-)-criocerine [9].

In our research project, we intended to investigate the DielsAlder reaction of vindoline (4) and its newly synthesized ortho-quinone derivative [10].

\section{Results and discussion}

In spite of the fact that the $\mathrm{C}=\mathrm{C}$ double bond in the 14,15-position of vindoline (4) is not a dienophilic electron deficient structural part, based on the analogy that acrolein also exhibits this type of reactivity [10], we carried out its Diels-Alder reaction with methyl vinyl ketone as a "diene" in an attempt to obtain compound 5. However, a Friedel-Crafts reaction was observed to take place (Scheme 1) affording the alkylated adduct (6) in good yield (57\%). 
Next, the ortho-quinone derivative of vindoline (7) was treated unstable under the conditions of the reaction $\left(\right.$ at $\left.110^{\circ} \mathrm{C}\right)$ with $N$-phenylmaleimide in toluene in order to obtain adduct 8 and reacts further. On the one hand, the $(\mathrm{O}) \mathrm{C}-\mathrm{C}(\mathrm{O})$ bond in via the usual cycloaddition reaction. However, instead of this compound $\mathbf{8}$ is opened, a partial decarbonylation takes place adduct two by-products were isolated in low yields, a dimeric to furnish intermediate $\mathbf{C}$, and species 7 is acylated by the derivative (9) in a yield of $3 \%$, and a vindoline condensed with monoacyl intermediate $(\mathbf{C})$, resulting in compound $\mathbf{9}$. On the $N$-phenylmaleimide (10) in a yield of $2 \%$ (Scheme 2 ). other hand, the breaking of the $(\mathrm{O}) \mathrm{C}-\mathrm{C}(\mathrm{O})$ bond in compound

In the course of the reaction, the expected adduct (8) is $\mathbf{8}$ may be followed by a double decarbonylation to provide probably formed as an intermediate, as suggested by the species $\mathbf{B}$, as well as re-aromatization to form compound $\mathbf{1 0}$ relevant MS peak $(\mathrm{M}+\mathrm{H}=630)$. Compound 8 is probably (Scheme 3).

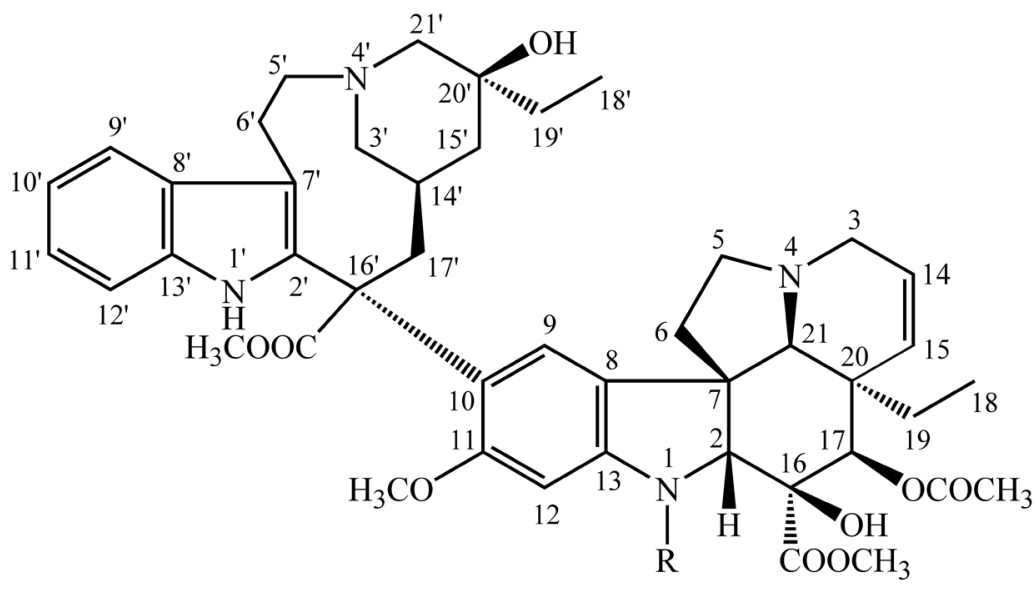

$1 \mathrm{R}=\mathrm{CH}_{3}$

$2 \mathrm{R}=\mathrm{CHO}$

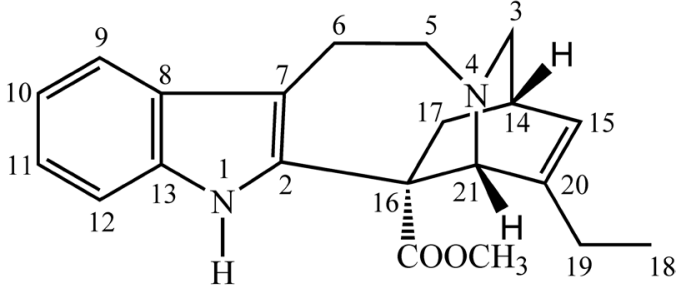

3

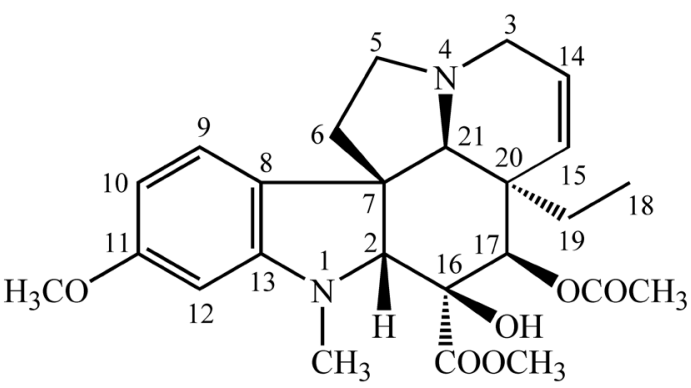

4

Fig. 1 The structure of Vinca alkaloids.<smiles>CC[C@]12C=CCN3CC[C@]4(c5ccc(OC)cc5N(C)[C@H]4[C@](O)(C(C)=O)[C@@H]1OC(C)=O)[C@@H]32</smiles><smiles>C=CC(CCCC(C)=O)C(C)C(=O)O</smiles>

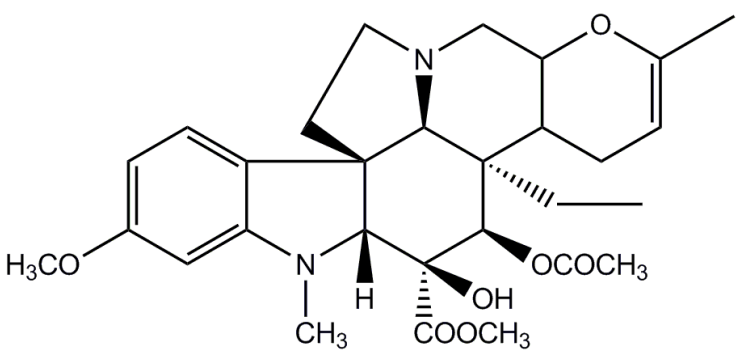
5<smiles>CC[C@]12C=CCN3CC[C@]4(c5cc(CCC(C)=O)c(OC)cc5N(C)[C@]4(C)[C@](O)(C(C)=O)[C@@H]1OC(C)=O)[C@@H]32</smiles>

6

Scheme 1 The Friedel-Crafts reaction observed. 


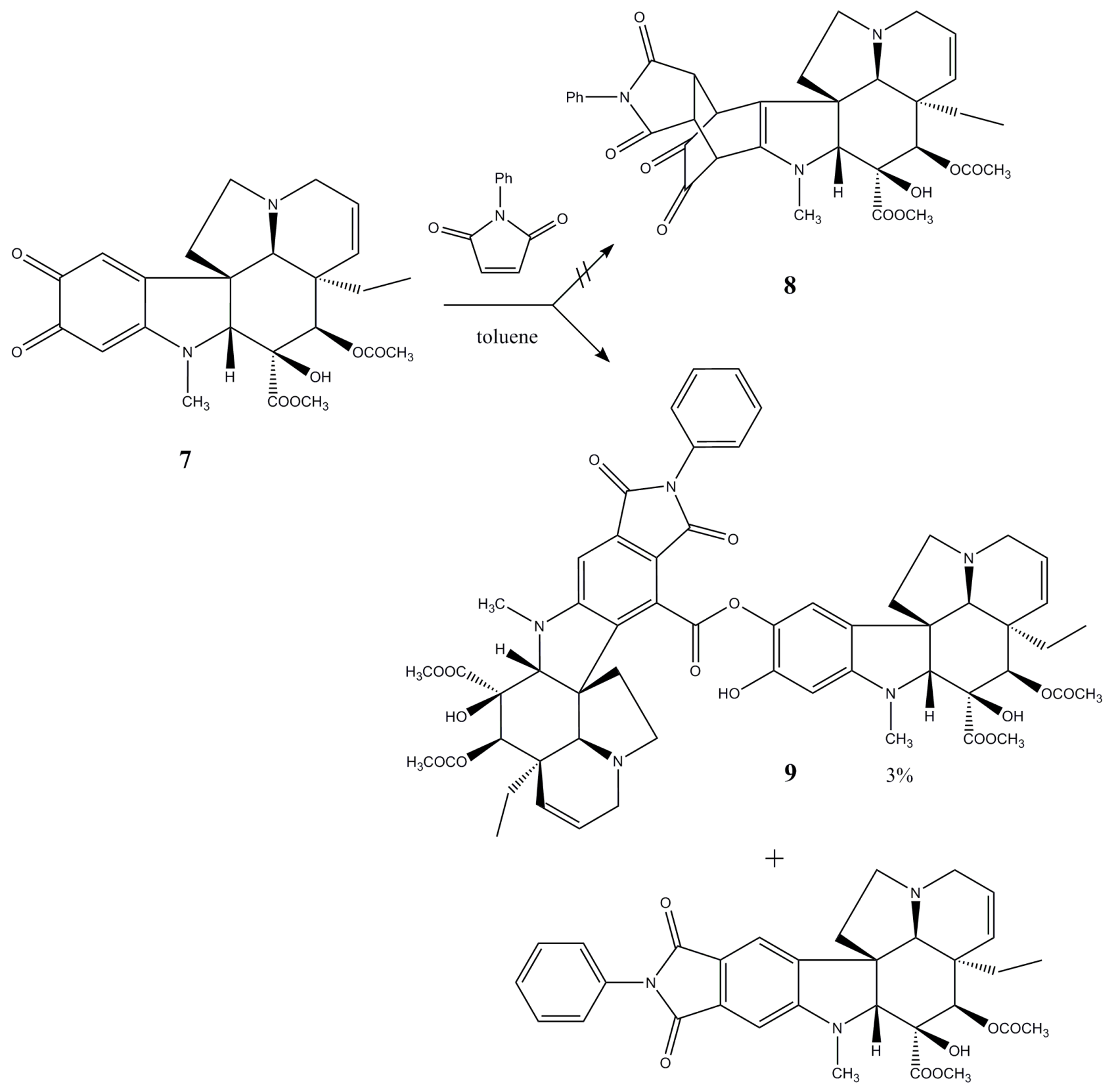

$10 \quad 2 \%$

Scheme 2 Outcome of the reaction between the ortho-quinone derivative of vindoline and $N$-phenylmaleimide

\section{Experimental}

\subsection{General}

Melting points were measured on a VEB Analytik Dresden PHMK-77/1328 apparatus and are uncorrected. IR spectra were recorded on Zeiss IR 75 and 80 instruments. NMR measurements were performed on a Varian $800 \mathrm{MHz}$ NMR spectrometer equipped with a ${ }^{1} \mathrm{H}\left\{{ }^{13} \mathrm{C} /{ }^{15} \mathrm{~N}\right\}$ Triple Resonance ${ }^{13} \mathrm{C}$ Enhanced Salt Tolerant Cold Probe operating at $799.7 \mathrm{MHz}$ for ${ }^{1} \mathrm{H}$ and $201.1 \mathrm{MHz}$ for ${ }^{13} \mathrm{C}$, and a Varian $500 \mathrm{MHz}$ NMR spectrometer equipped with a ${ }^{1} \mathrm{H}\left\{{ }^{13} \mathrm{C} /{ }^{15} \mathrm{~N}\right\} 5 \mathrm{~mm}$ PFG Triple Resonance ${ }^{13} \mathrm{C}$ Enhanced Cold Probe operating at $499.9 \mathrm{MHz}$ for ${ }^{1} \mathrm{H}$ and $125.7 \mathrm{MHz}$ for ${ }^{13} \mathrm{C}$. Chemical shifts are given on the delta scale as parts per million ( $\mathrm{ppm}$ ) with tetramethylsilane (TMS) $\left(0.00\right.$ ppm for ${ }^{1} \mathrm{H}$ and $\left.{ }^{13} \mathrm{C}\right) \cdot{ }^{1} \mathrm{H}-{ }^{1} \mathrm{H}$, direct ${ }^{1} \mathrm{H}-{ }^{13} \mathrm{C}$, and long-range ${ }^{1} \mathrm{H}-{ }^{13} \mathrm{C}$ scalar spin-spin connectivities were established from 2D zTOCSY, gHSQCAD, and gHMBCAD experiments, respectively. All pulse sequences were applied by using the standard spectrometer software package. All experiments were performed at $298 \mathrm{~K}$. HRMS and MS-MS analyses were performed on an LTQ FT Ultra (Thermo Fisher Scientific) system. The ionization method was ESI, operated in positive ion mode. The protonated molecular ion peaks were fragmented by CID at a normalized collision energy of $35 \%$. For the CID experiment helium was used as the collision gas. 
<smiles>[Y]C1C2=CC(=O)C(=O)C=C2N(C)C(C)(C)C1C</smiles>

7 $\underset{\begin{array}{l}\text { Diels-Alder } \\ \text { cycloaddition }\end{array}}{\stackrel{\mathrm{Ph}}{\mathrm{N}} Y^{\mathrm{O}}}$

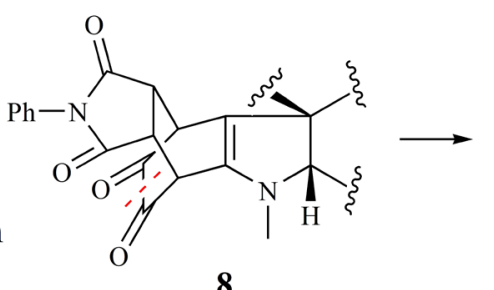

8

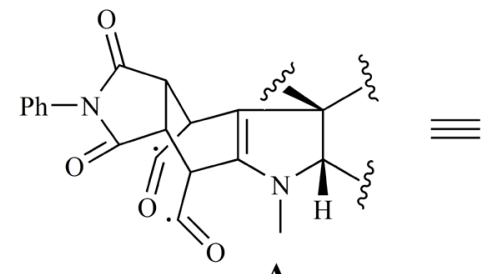

A

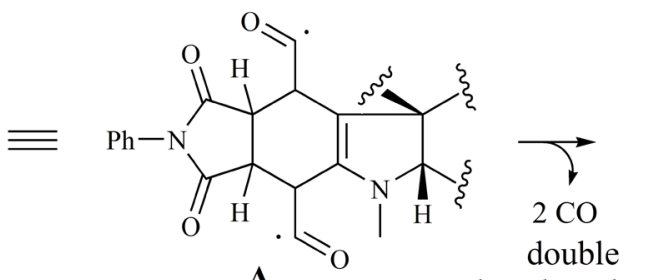

A decarbonylation

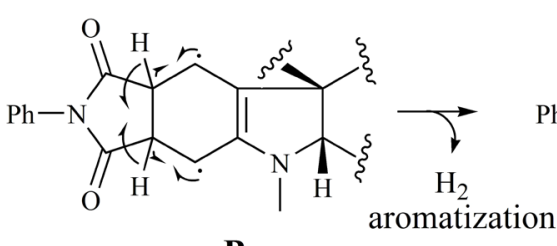

B<smiles>CCC1(C)c2cc3c(cc2N(C)[C@@H](C)[C@H]1C)C(=O)N(c1ccccc1)C3=O</smiles>

10

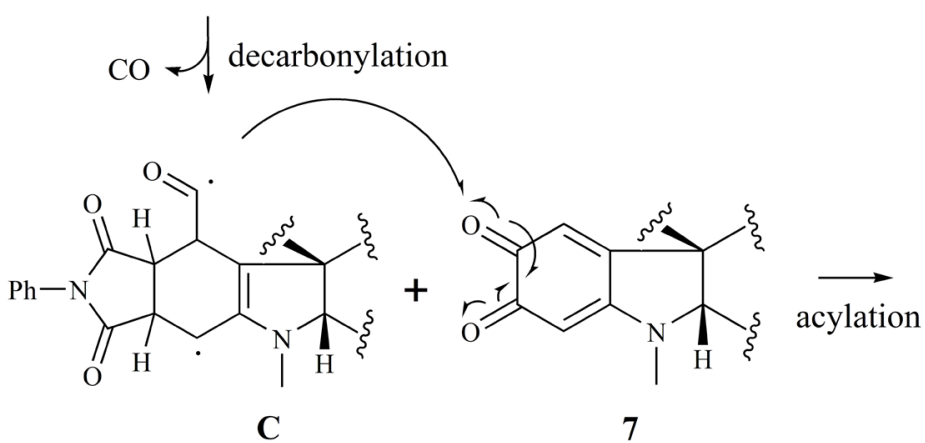

C

7

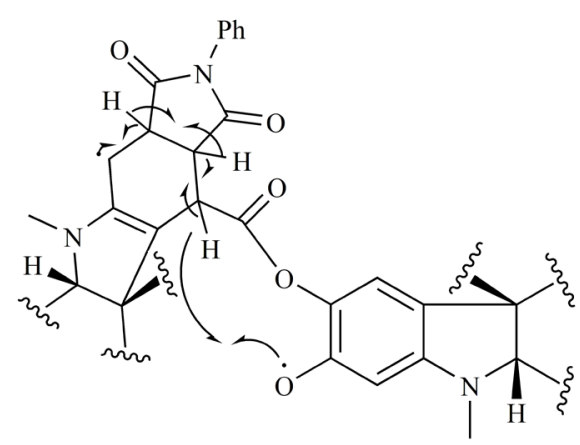

D

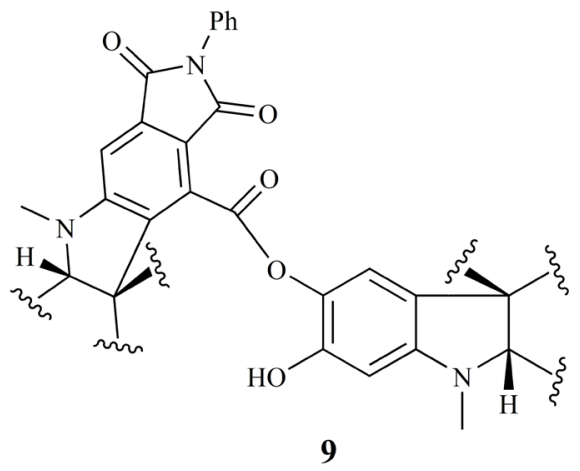

Scheme 3 The proposed mechanism for the reaction.

The samples were dissolved in methanol. Data acquisition and analysis were accomplished with Xcalibur software version 2.0 (Thermo Fisher Scientific). TLC was carried out using Kieselgel $60 \mathrm{~F}_{254}$ (Merck) glass plates.

\subsection{Friedel-Crafts reaction of vindoline (4)}

Vindoline (4) (400 mg, $0.876 \mathrm{mmol})$ was dissolved in dichloromethane $(40 \mathrm{ml})$, and methyl vinyl ketone $(0.7 \mathrm{ml}$, $8.76 \mathrm{mmol})$ and trifluoroacetic acid $(2.8 \mathrm{ml}, 36.8 \mathrm{mmol})$ were added at room temperature under stirring. After $24 \mathrm{~h}$ the reaction mixture was washed with $5 \%$ aqueous sodium bicarbonate $(40 \mathrm{ml})$ and the aqueous phase was extracted with dichloromethane $(2 \times 30 \mathrm{ml})$. The combined organic phase was dried (magnesium sulfate) and evaporated to dryness. The crude product was purified by preparative TLC (dichloromethanemethanol 15:1), and $264 \mathrm{mg}$ (57\%) of adduct 6 was obtained. Mp. $68^{\circ} \mathrm{C}$.

TLC: dichloromethane-methanol 20:1, $R_{f}=0.52$.

IR (KBr): 3469, 2934, 1713, 1369, 1241, $1042 \mathrm{~cm}^{-1}$.

${ }^{1} \mathrm{H}$ NMR $\left(799.7 \mathrm{MHz}\right.$, DMSO- $\left.d_{6}\right) \delta(\mathrm{ppm}) 0.40(3 \mathrm{H}, \mathrm{t}, J=7.5$ $\left.\mathrm{Hz}, \mathrm{H}_{3}-18\right), 0.89\left(1 \mathrm{H}, \mathrm{dq}, J=15.0,7.5 \mathrm{~Hz}, \mathrm{H}_{\mathrm{x}}-19\right), 1.46(1 \mathrm{H}$, dq, $\left.J=15.0,7.5 \mathrm{~Hz}, \mathrm{H}_{\mathrm{y}}-19\right), 1.93\left(3 \mathrm{H}, \mathrm{s}, \mathrm{C}(17)-\mathrm{OC}(\mathrm{O}) \mathrm{CH}_{3}\right)$, 
$2.06\left(3 \mathrm{H}, \mathrm{s}, \mathrm{CH}_{3} \mathrm{C}(\mathrm{O}) \mathrm{CH}_{2} \mathrm{CH}_{2}\right), 2.15-2.24\left(2 \mathrm{H}, \mathrm{m}, \mathrm{H}_{2}-6\right), 2.53-$ $2.69\left(9 \mathrm{H}, \mathrm{m}, \mathrm{CH}_{3} \mathrm{C}(\mathrm{O}) \mathrm{CH}_{2} \mathrm{CH}_{2}, \mathrm{~N}(1)-\mathrm{CH}_{3}, \mathrm{H}-21, \mathrm{H}_{\mathrm{x}}-5\right), 2.79$ (1H, ddd, $\left.J=16.2,2.3,1.7 \mathrm{~Hz}, \mathrm{H}_{\mathrm{x}}-3\right), 3.26-3.30\left(1 \mathrm{H}, \mathrm{m}, \mathrm{H}_{\mathrm{y}}-5\right)$, $3.42\left(1 \mathrm{H}\right.$, ddd, $\left.J=16.2,4.9,1.6 \mathrm{~Hz}, \mathrm{H}_{\mathrm{y}}-3\right), 3.49$ (1H, s, H-2), $3.65\left(3 \mathrm{H}, \mathrm{s}, \mathrm{C}(16)-\mathrm{COOCH}_{3}\right), 3.75\left(3 \mathrm{H}, \mathrm{s}, \mathrm{C}(11)-\mathrm{OCH}_{3}\right), 5.08$ (1H, ddd, $J=10.1,2.3,1.6 \mathrm{~Hz}, \mathrm{H}-15), 5.19$ (1H, s, H-17), 5.82 (1H, ddd, $J=10.1,4.9,1.7$ Hz, H-14), 6.29 (1H, s, H-12), 6.92 (1H, s, H-9), 8.80 (1H, br, C(16)-OH), ${ }^{13} \mathrm{C}$ NMR (201.1 MHz, DMSO- $\left.d_{6}\right) \delta(\mathrm{ppm}) 7.4(\mathrm{C}-18), 20.6\left(\mathrm{C}(17)-\mathrm{OC}(\mathrm{O}) \mathrm{CH}_{3}\right), 23.9$ $\left(\mathrm{CH}_{3} \mathrm{C}(\mathrm{O}) \mathrm{CH}_{2} \mathrm{CH}_{2}\right), 29.5\left(\mathrm{CH}_{3} \mathrm{C}(\mathrm{O}) \mathrm{CH}_{2} \mathrm{CH}_{2}\right), 30.3(\mathrm{C}-19), 38.7$ $\left(\mathrm{N}(1)-\mathrm{CH}_{3}\right), 42.3(\mathrm{C}-20), 43.4\left(\mathrm{CH}_{3} \mathrm{C}(\mathrm{O}) \mathrm{CH}_{2} \mathrm{CH}_{2}\right), 43.6(\mathrm{C}-6)$, 50.4 (C-3), $51.2(\mathrm{C}-5), 51.6\left(\mathrm{C}(16)-\mathrm{COOCH}_{3}\right), 52.2(\mathrm{C}-7), 55.3$ $\left(\mathrm{C}(11)-\mathrm{OCH}_{3}\right), 66.0$ (C-21), 75.9 (C-17), 78.6 (C-16), 83.0 (C-2), 93.6 (C-12), 119.5 (C-10), 123.4 (C-9), 124.0 (C-8), 124.3 (C-14), 129.9 (C-15), 151,7 (C-13), 157.7 (C-11), 170.0 $\left(\mathrm{C}(17)-\mathrm{OC}(\mathrm{O}) \mathrm{CH}_{3}\right), 171.6\left(\mathrm{C}(16)-\mathrm{COOCH}_{3}\right), 208.0\left(\mathrm{CH}_{3} \mathrm{C}(\mathrm{O})\right.$ $\mathrm{CH}_{2} \mathrm{CH}_{2}$ ).

HRMS: $\quad \mathrm{M}+\mathrm{H}=527.27615 \quad\left(\mathrm{C}_{29} \mathrm{H}_{39} \mathrm{~N}_{2} \mathrm{O}_{7} ;\right.$ delta=1.8 ppm); HR-ESI-MS-MS (CID=35\%; rel. int. \%): 509(13); 467(100); 435(3); 407(2); 258(9).

\subsection{Reaction of ortho-quinone derivative of vindoline (7) and $N$-phenylmaleimide}

The quinone derivative 7 (102 $\mathrm{mg}, 0.223 \mathrm{mmol})$ was dissolved in toluene $(10 \mathrm{ml})$, then $N$-phenylmaleimide (43 mg, $0.248 \mathrm{mmol}$ ) was added. The reaction mixture was refluxed for $42 \mathrm{~h}$. The solvent was evaporated, and the residue was separated by preparative TLC (dichloromethane-methanol 20:1). Two products were isolated, dimer $9(6 \mathrm{mg}, 3 \%)$ and the condensed monomer 10 (3 mg, 2\%).

Dimer derivative 9: Mp. $195^{\circ} \mathrm{C}$ (decomp.).

TLC: dichloromethane-methanol 20:1, $R_{f}=0.39$.

IR (KBr): 3401, 2929, 1747, 1715, 1587, 1385, $1234 \mathrm{~cm}^{-1}$.

${ }^{1} \mathrm{H}$ NMR (499.9 MHz, $\mathrm{CDCl}_{3}$ ) $\delta$ (ppm) 0.47 (3H, t, $J=7.4$ $\left.\mathrm{Hz}, \mathrm{H}_{3}-18^{\prime}\right), 0.58\left(3 \mathrm{H}, \mathrm{t}, J=7.4 \mathrm{~Hz}, \mathrm{H}_{3}-18\right), 1.17-1.25(2 \mathrm{H}$, m, $\left.\mathrm{H}_{\mathrm{x}}-19^{\prime}, \mathrm{H}_{\mathrm{x}}-19\right), 1.69-1.77$ (1H, m, $\left.\mathrm{H}_{\mathrm{y}}-19^{\prime}\right), 1.79-1.88$ (1H, m, H -19$), 2.07$ (3H, s, C(17')-OC(O)CH $), 2.10$ (3H, s, $\left.\mathrm{C}(17)-\mathrm{OC}(\mathrm{O}) \mathrm{CH}_{3}\right), 2.15-2.20\left(1 \mathrm{H}, \mathrm{m}, \mathrm{H}_{\mathrm{x}}-6\right.$ '), $2.38-2.61(5 \mathrm{H}$, m, $\left.\mathrm{H}_{\mathrm{x}}-5^{\prime}, \mathrm{H}_{\mathrm{y}}-6^{\prime}, \mathrm{H}-21^{\prime}, \mathrm{H}_{\mathrm{x}}-5, \mathrm{H}_{\mathrm{x}}-6\right), 2.66$ (3H, s, N(1')-CH $\mathrm{H}_{3}$, 2.75-2.82 (1H, m, $\mathrm{H}_{\mathrm{x}}-3$ ') $, 2.84-2.88\left(1 \mathrm{H}, \mathrm{m}, \mathrm{H}_{\mathrm{y}}-6\right), 2.88(3 \mathrm{H}$, $\left.\mathrm{s}, \mathrm{N}(1)-\mathrm{CH}_{3}\right), 2.91-2.97\left(1 \mathrm{H}, \mathrm{m}, \mathrm{H}_{\mathrm{x}}-3\right), 3.35-3.58(5 \mathrm{H}, \mathrm{m}$, $\left.\mathrm{H}_{\mathrm{y}}-5, \mathrm{H}_{\mathrm{y}}-5^{\prime}, \mathrm{H}-21, \mathrm{H}_{\mathrm{y}}-3, \mathrm{H}_{\mathrm{y}}-3^{\prime}\right), 3.77$ (1H, s, H-2'), $3.80(3 \mathrm{H}$, s, C(16')- $\left.\mathrm{COOCH}_{3}\right), 3.85\left(3 \mathrm{H}, \mathrm{s}, \mathrm{C}(16)-\mathrm{COOCH}_{3}\right), 4.15(1 \mathrm{H}$, s, H-2), 5.19-5.23 (2H, m, H-15, H-15'), 5.48 (1H, s, H-17), 5.50 (1H, s, H-17'), 5.82-5.86 (2H, m, H-14, H-14'), 6.23 (1H, s, H-12'), 6.51 (1H, br s, H-9'), 7.07 (1H, s, H-12), 7.39-7.45 (3H, m, H-4", H-2", H-6"), 8.44 (1H, br s, C(11')-OH), 9.010.2 (2H, br, C(16)-OH), C(16')-OH). ${ }^{13} \mathrm{C} \mathrm{NMR} \mathrm{(201.1} \mathrm{MHz,}$ $\left.\mathrm{CDCl}_{3}\right) \delta(\mathrm{ppm}) 8.0$ (C-18'), 8.1 (C-18), 20.97 (C(17)-OC(O) $\left.\mathrm{CH}_{3}\right), 21.04$ (C(17')-OC(O)CH$\left.{ }_{3}\right), 31.0$ (C-19'), 32.0 (C-19), $36.7\left(\mathrm{~N}(1)-\mathrm{CH}_{3}\right), 38.2\left(\mathrm{~N}\left(1^{\prime}\right)-\mathrm{CH}_{3}\right), 43.1$ (C-20'), 43.2 (br, C-6), 43.5 (C-20), 44.1 (br, C-6'), 51.2 (C-3), 51.3 (C-3'), 52.4
(C(16')- $\left.\mathrm{COOCH}_{3}\right), 52.5$ (C-5), 52.6 (C-5'), 52.7 (C-7'), 52.8 $\left(\mathrm{C}(16)-\mathrm{COOCH}_{3}\right), 53.6$ (C-7), 66.5 (br, C-21), 67.9 (br, C-21'), 75.3 (br, C-17), 76.1 (br, C-17'), 78.4 (C-16), 79.3 (C-16'), 83.2 (br, C-2'), 83.4 (br, C-2), 99.2 (C-12'), 104.0 (C-12), 114.7 (C-9'), 117.3 (br, C-10), 123.9 (br, C-14), 124.0 (br, C-14'), 124.9 (C-8'), 125.8 (C-9), 126.6 (C-2”, C-6”), 128.4 (C-4"), 129.1 (C-3", C-5”), 129.9 (C-10'), 130.0 (C-15), 130.4 (C-15'), 131.1 (C-1”), 135.2 (C-11), 136.6 (br, C-8), 149.9 (C-11'), 152.4 (C-13'), 159.2 (C-13), 164.9* (C(9)-COOAr), $165.9(\mathrm{C}(11)-C \mathrm{O}), 167.6^{*}(\mathrm{C}(10)-\mathrm{CO}), 170.8$ (C(17)-OC(O) $\left.\mathrm{CH}_{3}, \mathrm{C}\left(17^{\prime}\right)-\mathrm{OC}(\mathrm{O}) \mathrm{CH}_{3}\right), \quad 171.7 \quad\left(\mathrm{C}(16)-\mathrm{COOCH}_{3}\right), \quad 171.9$ $\left(\mathrm{C}\left(16^{\prime}\right)-\mathrm{COOCH}_{3}\right)$. *: uncertain assignment

HRMS: $\quad \mathrm{M}+\mathrm{H}=1056.42448 \quad\left(\right.$ delta $=0.8 \mathrm{ppm} ; \quad \mathrm{C}_{57} \mathrm{H}_{62} \mathrm{O}_{15} \mathrm{~N}_{5}$ ). HR-ESI-MS-MS (CID=35\%, rel. int. \%): 1038(12); 996(100); 978(3); 894(10); 787(8); 598(53).

Condensed monomer 10: Mp. $120{ }^{\circ} \mathrm{C}$.

TLC: dichloromethane-methanol 20:1, $R_{f}=0.58$.

IR (KBr): 3468, 2929, 1745, 1713, 1372, 1236, $754 \mathrm{~cm}^{-1}$.

${ }^{1} \mathrm{H}$ NMR (499.9 MHz, $\left.\mathrm{CDCl}_{3}\right) \delta(\mathrm{ppm}) 0.56(3 \mathrm{H}, \mathrm{t}, J=7.4$ $\left.\mathrm{Hz}, \mathrm{H}_{3}-18\right), 1.11\left(1 \mathrm{H}, \mathrm{dq}, J=14.4,7.4 \mathrm{~Hz}, \mathrm{H}_{\mathrm{x}}-19\right), 1.71(1 \mathrm{H}$, $\left.\mathrm{dq}, J=14.4,7.4 \mathrm{~Hz}, \mathrm{H}_{\mathrm{y}}-19\right), 2.09\left(3 \mathrm{H}, \mathrm{s}, \mathrm{C}(17)-\mathrm{OC}(\mathrm{O}) \mathrm{CH}_{3}\right)$, 2.31-2.39 (1H, m, $\left.\mathrm{H}_{\mathrm{x}}-6\right), 2.40-2.50\left(1 \mathrm{H}, \mathrm{m}, \mathrm{H}_{\mathrm{y}}-6\right), 2.63-2.68$ $\left(1 \mathrm{H}, \mathrm{m}, \mathrm{H}_{\mathrm{x}}-5\right), 2.85\left(1 \mathrm{H}\right.$, br s, H-21), $2.86\left(3 \mathrm{H}, \mathrm{s}, \mathrm{N}(1)-\mathrm{CH}_{3}\right)$, 2.87-2.93 (1H, m, $\left.\mathrm{H}_{\mathrm{x}}-3\right), 3.48-3.61\left(2 \mathrm{H}, \mathrm{m}, \mathrm{H}_{\mathrm{y}}-3, \mathrm{H}_{\mathrm{y}}-5\right), 3.83$ $\left(3 \mathrm{H}, \mathrm{s}, \mathrm{C}(16)-\mathrm{COOCH}_{3}\right), 4.04(1 \mathrm{H}$, br s, H-2), 5.27-5.31 (1H, $\mathrm{m}, \mathrm{H}-15), 5.39(1 \mathrm{H}, \mathrm{s}, \mathrm{H}-17), 5.91(1 \mathrm{H}, \mathrm{ddd}, J=10.3,5.0$, $1.4 \mathrm{~Hz}, \mathrm{H}-14), 6.94$ (1H, s, H-12), 7.36-7.40 (1H, m, H-4'), $7.41-7.45$ (2H, m, H-2', H-6'), 7.47-7.52 (2H, m, H-3', H-5'), 7.56 (1H, s, H-9), 9.35 (1H, br, C(16)-OH). ${ }^{13} \mathrm{C}$ NMR $\left(125.7 \mathrm{MHz}, \mathrm{CDCl}_{3}\right) \delta(\mathrm{ppm}) 7.7$ (C-18), 20.9 (C(17)-OC(O) $\left.\mathrm{CH}_{3}\right), 31.2(\mathrm{C}-19), 36.6\left(\mathrm{~N}(1)-\mathrm{CH}_{3}\right), 43.0(\mathrm{C}-20), 44.4$ (C-6), 50.9 (C-3), 51.8 (C-5), $52.6\left(\mathrm{C}(16)-\mathrm{COOCH}_{3}\right) .52 .9(\mathrm{C}-7)$, 67.4 (C-21), 75.7 (C-17), 79.1 (C-16), 82.6 (br, C-2), 102.4 (C-12), 118.2 (C-9), 121.5 (C-10), 124.3 (br, C-14), 126.5 (C-2', C-6'), 127.8 (C-4'), 129.1 (C-3', C-5'), 129.9 (C-15), 132.0 (C-1'), 135.0 (C-11), 139.1 (C-8), 157.5 (C-13), 167.26 $(\mathrm{C}(11)-\mathrm{CO}), 167.30(\mathrm{C}(10)-\mathrm{CO}), 170.6\left(\mathrm{C}(17)-\mathrm{OC}(\mathrm{O}) \mathrm{CH}_{3}\right)$ $171.4\left(\mathrm{C}(16)-\mathrm{COOCH}_{3}\right)$.

HRMS: $\mathrm{M}+\mathrm{H}=572.23887 \quad\left(\right.$ delta $\left.=-0.5 \mathrm{ppm} ; \quad \mathrm{C}_{32} \mathrm{H}_{34} \mathrm{O}_{7} \mathrm{~N}_{3}\right)$. HR-ESI-MS-MS (CID=35\%, rel. int. \%): 554(10); 530(3); 512(100); 480(23); 452(6); 424(3); 303(9).

\section{References}

[1] Brossi, A., Suffness, M. (eds.) "Antitumor Bisindol Alkaloids from Catharanthus Roseus (L.)." In: The Alkaloids. Academic Press Inc., New York, USA, 37, pp. 1-240. 1990.

[2] Sisodiya, P. S. "Plant derived anticancer agents: A review." International Journal of Research and Development in Pharmacy \& Life Sciences. 2, pp. 293-308. 2013.

[3] Bölcskei, H., Szabó, L., Szántay, Cs. "Synthesis of vinblastine derivatives." Frontiers in Natural Product Chemistry. 1, pp. 43-49. 2005. https://doi.org/10.2174/1574089054583849 
[4] Keglevich, P., Hazai, L., Kalaus, Gy., Szántay, Cs. "Modifications on the Basic Skeletons of Vinblastine and Vincristine." Molecules. 17, pp. 5893-5914. 2012.

https://doi.org/10.3390/molecules17055893

[5] Campbell, E. R., Skepper, C. K., Sankar, K., Duncan, K. K., Boger, D. L. "Transannular Diels-Alder/1,3-dipolar cycloaddition cascade of 1,3,4-oxadiazoles: total synthesis of a unique set of vinblastine analogues." Organic Letters. 15, pp. 5306-5309. 2013. https://doi.org/10.1021/o1402549n

[6] Horning, B. D., MacMillan, D. W. C. "Nine-step enantioselective total synthesis of (-)-vincorine." Journal of the American Chemical Society. 135, pp. 6442-6445. 2013.

https://doi.org/10.1021/ja402933s

[7] Jones, S. B., Simmons, B., MacMillan, D. W. C. "Nine-step enantioselective total synthesis of (+)-minfiensine." Journal of the American Chemical Society. 131, pp. 13606-13607. 2009.

https://doi.org/10.1021/ja906472m
[8] Yuan, Z. Q., Ishikawa, H., Boger, D. L. "Total synthesis of natural (+)[corrected] and ent-(-)-4-desacetoxy-6,7-dihydrovindorosine [corrected] and natural and ent-minovine: oxadiazole tandem intramolecular Diels-Alder/1,3-dipolar cycloaddition reaction." Organic Letters. 7, pp. 741-744. 2005.

https://doi.org/10.1021/o1050017s

[9] Szántay, Cs., Jr., Moldvai, I., Tárkányi, G., Szántay, Cs. "Synthesis of Vinca alkaloids and related compounds. 79. An intriguing retro Diels-Alder reaction." The Journal of Organic Chemistry. 61, pp. 2946-2950. 1996. https://doi.org/10.1021/jo9521110

[10] Keglevich, A., Mayer, Sz., Szigetvári, Á., Sánta, Zs., Dékány, M., Szántay, Cs., Jr., Keglevich, P., Hazai, L. "Attempted synthesis of Vinca alkaloids condensed with three-membered rings." To be published.

[11] Longley, Jr., R. I., Emerson, W. S. "The 1,4-addition of vinyl ethers to $\alpha, \beta$-unsaturated carbonyl compounds." Journal of the American Chemical Society. 72, pp. 3079-3081. 1950. https://doi.org/10.1021/ja01163a076 